\title{
FACTORES RELACIONADOS CON LA CALIDAD DE LA EDUCACIÓN SUPERIOR EN COLOMBIA
}

\section{FACTORS RELATED TO THE QUALITY OF HIGHER EDUCATION IN COLOMBIA}

\author{
Phd. Yolanda González-Castro ${ }^{\mathrm{a}}$, MSc. Marta Milena Peñaranda-Peñaranda ${ }^{\mathrm{b}}$, MSc. Omaira \\ Manzano-Durán ${ }^{\mathrm{c}}$ \\ ${ }^{a}$ Grupo de Investigación GRINDES. Docente UNAD. Pamplona-Colombia. \\ yolanda.gonzalez@unad.edu.co \\ ${ }^{\mathrm{b}}$ Grupo de Investigación GIDSE. Universidad Francisco de Paula Santander Ocaña-Colombia. \\ mmpenarandap@ufpso.edu.co \\ ${ }^{c}$ Grupo de Investigación GRINDES. Docente UNAD. Ocaña-Colombia. \\ omaira.manzano@unad.edu.co
}

Fecha de recepción: 10-04-2017

Fecha de aprobación: 15-06-2017

\begin{abstract}
Resumen: Esta investigación analiza los factores del entorno del estudiante como el estrato social, la edad y el número de horas dedicadas al trabajo que pueden influir en el resultado de las pruebas Saber Pro del Instituto Colombiano para el Fomento de la Educación Superior [IFCES] en las competencias genéricas: Lectura Crítica, Comunicación Escrita y Razonamiento Cuantitativo en los estudiantes del programa de Administración de Empresas.

La investigación es de tipo cuantitativo con diseño no experimental longitudinal. Las principales técnicas estadísticas de análisis utilizadas son: Medidas de tendencia central, varianza y regresión lineal. Los resultados indican que no hay una fuerte correlación entre el estrato, las horas dedicadas al trabajo, la edad y los resultados en las competencias genéricas anteriormente descritas. Sin embargo, la variable que explica mejor la variabilidad de los resultados en las competencias genéricas es la edad.
\end{abstract}

Palabras clave: Pruebas Saber Pro, competencias genéricas, lectura crítica, comunicación escrita, razonamiento cuantitativo

Abstract: This research analyzes the factors of the student's environment as the social stratum, the age and the number of hours dedicated to the work that can influence the results of the Saber 
Pro tests of the Colombian Institute for the Promotion of Higher Education (IFCES) in the Generic competences: Critical Reading, Written Communication and Quantitative Reasoning in students of the Business Administration program.

The research is of quantitative type with non-experimental longitudinal design. The main statistical techniques of analysis used are: Measures of central tendency, variance and linear regression. The results indicate that there is not a strong correlation between the stratum, the hours dedicated to work, the age and the results in the generic competences previously described. However, the variable that best explains the variability of the results in the generic competences is age.

Keywords: Tests Saber Pro, Generic Skills, Critical Reading, Written Communication, Quantitative Reasoning.

\section{INTRODUCCIÓN}

Las pruebas Saber Pro ${ }^{1}$ establecidas por el Ministerio de Educación Nacional de Colombia [MEN] para evaluar la calidad de la educación superior en Colombia se han constituido en uno de los mecanismos para evaluar el rendimiento académico de las universidades y las competencias de los estudiantes que cursan el último año de un programa de pregrado. Las pruebas evalúan competencias básicas o genéricas para todos los programas y competencias específicas por programas afines. Las competencias básicas o genéricas evaluadas son: Lectura Crítica, Comunicación Escrita, Razonamiento Cuantitativo, Competencias Ciudadanas e inglés.

Las Competencias Genéricas son transversales a las áreas curriculares y del conocimiento (disciplinas) de todos los programas de educación superior; se desarrollan a través del trabajo concreto en una o más áreas y pueden ser transferidas a otros contextos. Son longitudinales, pues están presentes en todos los niveles y su complejidad es creciente a lo largo de toda

\footnotetext{
${ }^{1}$ Op. Cit.
}

la trayectoria educativa. Deben ser desarrolladas por todas las personas, aunque no necesariamente en el mismo nivel. Se les llama también competencias del siglo XXI y son indispensables para el desempeño académico y laboral (ICFES, 2011).

La competencia genérica Lectura Crítica mide los desempeños asociados a la lectura, pensamiento crítico y entendimiento interpersonal: lo que significa, leer analítica y reflexivamente, comprender los planteamientos expuestos en un texto e identificar sus perspectivas y juicios de valor, identificar y recuperar información presente en uno o varios textos, construir su sentido global, establecer relaciones entre enunciados y evaluar su intencionalidad. La competencia genérica Razonamiento Cuantitativo se relaciona con la comprensión de conceptos básicos matemáticos e interpretación de los mismos, y la competencia genérica Comunicación Escrita, permite notificar ideas por escrito referidas a un tema dado, teniendo en cuenta la claridad de la estructura y la organización (ICFES, 2011).

La relevancia de este artículo de investigación científica está en la 
consideración de otros factores inherentes a las características del estudiante y su entorno como la edad, el estrato y las horas dedicadas al trabajo, para comprender su influencia en el rendimiento en la pruebas Saber Pro, convirtiéndose en una herramienta fundamental para direccionar políticas administrativas en el programa de Administración de Empresas que busquen el mejoramiento continuo de la calidad.

\section{METODOLOGÍA}

El estudio que se describe es ex post-facto o retrospectivo (Hernández, 2010. p.149) pues se realizó después de que los hechos ocurrieron, recolectando sus evidencias. Se trata de un trabajo con un enfoque cuantitativo y de tipo descriptivo y correlacional. A su vez, es un diseño de investigación no experimental, pues se consideran datos de una muestra que no fue planeada deliberadamente para ello. $\mathrm{Su}$ enfoque es igualmente longitudinal de tendencia pues se consideran datos de los años 2005 al 2011 de resultados de las pruebas Saber Pro en competencias genéricas para el programa de Administración de Empresas.

La población con la que se llevó a cabo la investigación son los estudiantes de los programas Administración de Empresas de la UNAD que presentaron las pruebas Saber Pro en los años mencionados, tomando la información necesaria de la base de datos del ICFES ${ }^{2}$, donde se ofrece un conjunto de indicadores agrupados por cada institución de educación superior, en este caso para los componentes evaluados en las pruebas genéricas Lectura Crítica, Comunicación Escrita y Razonamiento Cuantitativo, y la información correspondiente a edad, estrato y horas de trabajo del estudiante.

${ }^{2}$ Op. Cit
La muestra utilizada fue seleccionada a partir de la población de manera aleatoria, posteriormente se analizó la información utilizando el software SPSS. Los datos del estudio fueron analizados mediante las siguientes técnicas estadísticas: frecuencias, medidas de tendencia central y medidas de variabilidad, y análisis de regresión lineal.

Este trabajo se desarrolló en tres fases; en la primera, se realizó una revisión de la literatura existente en cuanto a la relevancia de las pruebas Saber Pro para medir la calidad educativa y los elementos del entorno que influyen en dicho rendimiento; en la segunda, se analizan los datos empleando el software SPSS y se realiza un análisis de regresión lineal a las características del estudiante y su entorno, que pueden influir en los resultados de las pruebas Saber Pro competencias genéricas y finalmente se presentan los resultados con sus respectivas conclusiones y recomendaciones.

Se consideró como variable dependiente las características del estudiante y su entorno como edad, estrato y horas de trabajo, que parecen influir en los resultados de las pruebas Saber Pro en las competencias genéricas mencionadas, para lo cual se realizó un análisis de Regresión lineal.

\section{RESULTADOS}

\subsection{Resultados de la Revisión Teórica}

Bogoya (2012), manifiesta: "Si bien el aprendizaje alcanzado en una institución de educación superior podría estar signado por las condiciones socio económicas y académicas que los estudiantes demuestran al inicio de sus estudios, siempre será posible pensar en construir y desplegar 
estrategias pedagógicas para lograr que los estudiantes con mayores dificultades venzan las barreras propias del entorno y también puedan desarrollar altos niveles de comprensión".

Bogoya (2012), muestra indicadores por institución y para el país, con el fin de facilitar una comparación directa y encontrar la localización relativa de cada proyecto educativo en el ámbito nacional.

Se espera que los centros educativos den cuenta de la inversión de sus recursos con resultados de eficiencia; para ello se utilizan técnicas matemáticas $\mathrm{o}$ indicadores de desempeño. Hoy en día existe la comparación entre resultados de Pruebas de Estado como una medida de la eficiencia, al igual que se considera una relación directa entre eficiencia en los resultados como un indicador de calidad académica. No obstante Ciertos autores critican este tipo de análisis pues advierten que para este caso las unidades observables no son homogéneas (Díez, 2005).

Así, adoptar programas de medición y gestión de los resultados académicos en las universidades constituye una alternativa para potenciar sus resultados y cómo las universidades dan respuesta a los actuales retos del mundo moderno (Rivero, Vega, y Balagué, 2005).

El informe Coleman, mencionado por Chica y Ramírez (2009), examina para Estados Unidos el efecto de los insumos escolares sobre las diferencias en el desempeño escolar. Los resultados hallados en este estudio causaron controversia en el mundo académico, ya que el efecto encontrado fue prácticamente nulo, destacando que las diferencias en los resultados escolares se debían principalmente al origen social del estudiante.
Jencks (1972), realizó un trabajo similar en el cual se reafirmaron los resultados encontrados por Coleman (1966), su principal conclusión era que variables como los recursos financieros de la escuela, sus políticas y las características de los maestros, influyen de manera secundaria en la explicación del rendimiento escolar. En un caso más específico, Alexander y Simmons (1975), examinaron los determinantes del desempeño académico en países del Tercer Mundo, concluyendo que las características asociadas al entorno socioeconómico de los estudiantes eran las más relevantes, confirmando la hipótesis planteada por Coleman (1966) y Jencks (1972).

Heyneman (1976;1980) (2005) y sus colaboradores (Heyneman y Loxley,1982), citados por Cervini, basado en la revisión crítica de los estudios realizados con anterioridad y en sus propias investigaciones, cuestionaron la validez de la conclusión del informe Coleman debido a los errores de medición, a procedimientos metodológicamente inadecuados y a la incorrecta especificación de los modelos estadísticos utilizados. Los trabajos de Heynneman y de otros autores (Theisen et.alli,1983) tendían a advertir que el impacto de cada una de las variables endógenas y exógenas varía de acuerdo al nivel de desarrollo económico de los países en los que fueron realizados los estudios, es decir, "cuanto más industrializada es la sociedad, mayor será la probabilidad de que el rendimiento escolar sea afectado por el ambiente socio-económico del alumno y otras variables no escolares" (Heyneman,1976, p.205 citado por Cervini, 2005).

Respecto al rendimiento académico de las personas que trabajan es importante señalar 
como quienes tienen relación con la vida laboral desarrollan destrezas para la toma de decisiones y las capacidades generales de razonamiento (Denegri, Marianela; Del Vallle, Carlos; Gempp, $\mathrm{R}$ y Lara, M, 2006).

Cervini (2005), plantea que en realidad, la divergencia Coleman-Heyneman se podría explicar por las diferencias del comportamiento contextual de las mediciones utilizadas. En los países más desarrollados, altamente estratificados, es más fácil medir el 'estatus familiar' con alta discriminación, aumentando así la probabilidad de contribución explicativa respecto al logro escolar; los indicadores del sistema educativo, en cambio, son más homogéneos. En los países del Tercer Mundo, la situación más probable es exactamente la inversa. Los indicadores tradicionales de status social captan parcialmente las diferencias sociales, especialmente cuando se incluye sólo el aspecto material en la medición de clase social, dejando afuera aspectos culturales. Si estos aspectos se tienen en cuenta, se afirma, el efecto del origen social sobre el rendimiento se torna mayor (Lockheed, Fuller y Nyirondo, 1989 citados por Cervini, 2005). Por otra parte, y contrariamente a los países centrales, los sistemas educativos son más heterogéneos y segmentados.

En cuanto a la edad y el rendimiento académico las investigaciones permiten evidenciar como que el envejecimiento efectivamente conlleva cambios en el desempeño lingüístico. Este cambio se manifiesta principalmente en el enlentecimiento de los procesos al igual que se vincula el envejecimiento con la declinación de la memoria y al procesamiento del lenguaje. Por otra parte, el factor gramatical afecta el tiempo de procesamiento de ciertas palabras críticas y el tiempo de respuesta a preguntas sobre el contenido de las oraciones. (Riffo, B, Benotil, C, 2007).

\subsection{Análisis de Regresión Lineal}

Con el propósito de validar la siguiente hipótesis: "Existe una alta correlación entre el número de horas dedicadas al trabajo por el estudiante de Administración de Empresas y los resultados de la prueba Saber Pro en competencias genéricas", se procedió a realizar un análisis de regresión lineal que arrojó los siguientes resultados: Un coeficiente de correlación del $11.4 \%$ que indica la asociación entre las dos variables y un coeficiente de determinación del 1.3\% con la competencia genérica Lectura Crítica, lo cual nos indica que solo el $1.3 \%$ de la variación en los resultados se explica por la variación en el número de horas empleadas por los estudiantes en el trabajo. Los datos anteriores nos permiten negar la hipótesis inicialmente formulada.

De igual forma se realizó el análisis con la competencia genérica Comunicación Escrita para el mismo programa Administración de Empresas, arrojando un coeficiente de determinación del $3.2 \%$, es decir, solo el $3.2 \%$ de la variación en los resultados en la competencia genérica Comunicación Escrita es explicado por la variación en el número de horas laboradas, y por último el análisis de la competencia genérica Razonamiento Cuantitativo vs el número de horas de trabajo del estudiante del mismo programa Administración de Empresas que arrojó un coeficiente de correlación del $1.2 \%$ lo cual nos indica que el porcentaje de la variación total de la variable dependiente que se explica es muy bajo. Rechazando la hipótesis inicial.

Posteriormente se procedió a validar la siguiente hipótesis: "Existe una correlación 
entre la edad del estudiante del programa Administración de Empresas y los resultados en la pruebas Saber Pro en competencias genéricas" para lo cual se realizó un análisis de correlación que arrojó lo siguientes resultados (ver tabla 1).

Tabla 1. Correlación entre edad y resultados competencias genéricas para el programa Administración de Empresas de la UNAD, mediante el software SPSS.

Podemos apreciar en los resultados que la variable "edad" tiene una mayor correlación que la variable "horas de trabajo" en los resultados de las diferentes competencias genéricas; por ejemplo el $13 \%$ de la variación en los resultados de la prueba genérica Razonamiento Cuantitativo es explicado por la variación en la "edad". Sin embargo aún nos encontramos en un bajo nivel de correlación con todas las competencias genéricas respecto de las dos variables "edad" y "horas de trabajo".

De igual forma se procedió con la variable "estrato" (ver tabla 2) la cual también arrojó una muy baja correlación con las competencias genéricas, así:

Tabla 2. Correlación entre estrato y los resultados de las pruebas genéricas. Mediante el software SPSS

\begin{tabular}{|l|l|l|l|}
\hline Competencia & $R$ & R Square & $\begin{array}{l}\text { Std. Error of } \\
\text { the Estimate }\end{array}$ \\
\hline Lectura critica &, $069^{\mathrm{a}}$ &, 005 &,- 014 \\
\hline $\begin{array}{l}\text { Comunicación } \\
\text { Escrita }\end{array}$ &, $036^{\mathrm{a}}$ &, 001 &,- 019 \\
\hline $\begin{array}{l}\text { Razonamiento } \\
\text { cuantitativo }\end{array}$ &, $065^{\mathrm{a}}$ &, 004 &,- 015 \\
\hline
\end{tabular}

Muchos estudios, tanto a nivel nacional como internacional, han puesto en evidencia que las variables socioeconómicas juegan un papel primordial en la calidad de la educación medida a través del desempeño en pruebas estándar (Chica, S; Galvis, D y Ramírez, A 2009). En el resultado de esta investigación podemos apreciar que en el programa de Administración de Empresas no se observa un resultado superior que se pueda atribuir al estrato del estudiante.

Por otra parte, muchas investigaciones han arrojado que entre más edad implica una mayor probabilidad de ubicarse en un

\begin{tabular}{|l|l|l|l|}
\hline Competencia & R & R Square & $\begin{array}{l}\text { Std. Error of the } \\
\text { Estimate }\end{array}$ \\
\hline Lectura critica &, 297 &, 088 &, 81870 \\
\hline $\begin{array}{l}\text { Comunicación } \\
\text { Escrita }\end{array}$ &, 356 &, 127 &, 84532 \\
$\begin{array}{l}\text { Razonamiento } \\
\text { cuantitativo }\end{array}$ &, 365 &, 133 &, 84449 \\
\hline
\end{tabular}

rendimiento bajo en la pruebas (Chica, $\mathrm{S}$; Galvis, D y Ramírez, A 2009), en la presente investigación se pudo determinar que de las variables analizadas la que más influye en los resultados es la edad con una relación inversa, es decir a mayor edad menor puntaje en las pruebas.

Al efectuar un análisis de regresión lineal múltiple en el promedio obtenido en las pruebas genéricas se obtuvieron los siguientes resultados:

Tabla 3. Regresión lineal múltiple. Mediante el software SPSS

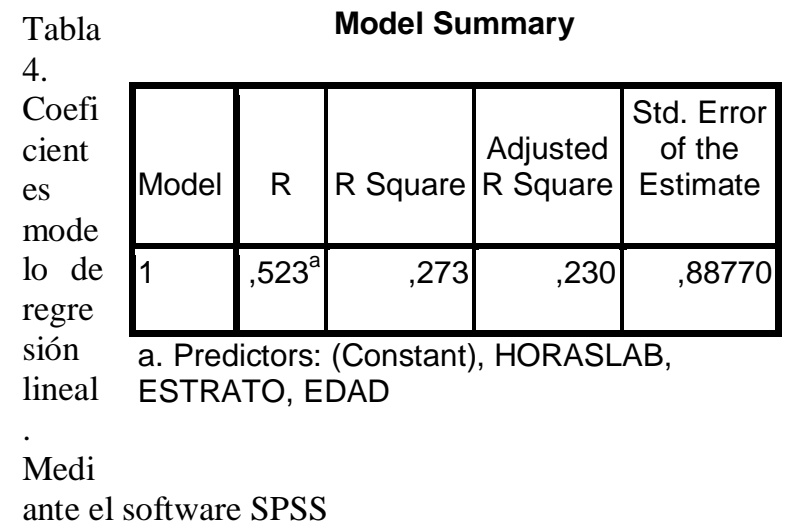




\section{Coefficients $^{\mathrm{a}}$}

\begin{tabular}{|l|r|r|r|r|r|}
\hline \multirow{2}{*}{ Model } & \multicolumn{2}{|c|}{$\begin{array}{l}\text { Unstandardized } \\
\text { Coefficients }\end{array}$} & $\begin{array}{c}\text { Stand. } \\
\text { Coef. }\end{array}$ & & \\
\cline { 2 - 4 } & \multicolumn{1}{|c|}{$\mathrm{B}$} & \multicolumn{1}{|c|}{$\begin{array}{c}\text { Std. } \\
\text { Error }\end{array}$} & Beta & \multicolumn{1}{c|}{$\mathrm{t}$} & Sig. \\
\hline EDAD &,- 047 &, 015 &,- 393 & $-3,03$ &, 004 \\
ESTRATO &,- 290 &, 165 &,- 223 & $-1,75$ &, 085 \\
HORASLAB &, 029 &, 014 &, 261 & 2,11 &, 039 \\
\hline
\end{tabular}

a. Dependent Variable: COMPGENERICS

Como se puede apreciar en las tablas 3 y 4 existe un coeficiente de correlación del $52.3 \%$, y de determinación del $27.3 \%$ lo que indica que el $27.3 \%$ de la variación en los resultados de las pruebas genéricas es explicado por las variables: Edad, estrato y horas de trabajo. Además los valores de significancia por debajo del $5 \%$ indican que la "edad" y las "horas laborales" son las variables que más influyen en este resultado.

\section{CONCLUSIONES}

La hipótesis inicial dada en cuanto a que las "horas dedicadas al trabajo" por parte del estudiante tienen una alta correlación con las competencias genéricas lectura crítica, comunicación escrita, y razonamiento cuantitativo fue rechazada para los resultados de cada una de las competencias mencionadas, lo mismo ocurre para la hipótesis que relaciona que un mayor estrato social repercute en un mayor rendimiento, lo cual se aleja de las conclusiones del informe Coleman que correlaciona de manera significativa el rendimiento con el nivel socioeconómico en países desarrollados; asimismo de las conclusiones de Cervini (2005), quien plantea que en realidad la divergencia con Coleman se debe a que los indicadores con que se miden las diferencias sociales en los países subdesarrollados solo tienen en cuenta aspectos materiales y se excluyen los aspectos culturales que podrían generar mayores diferencias en los resultados de rendimiento. Esto podría ser objeto de un estudio posterior a partir de modelos alternativos para la medición del estrato socioeconómico.

En el análisis de regresión lineal múltiple se pudo determinar que de las variables que caracterizan al estudiante y su entorno, la "edad" y las "horas laborales" son las variables que más influyen en los resultados. Lo anterior es un aporte investigativo a las reflexiones sobre la influencia del entorno del estudiante del programa Administración de Empresas en el rendimiento académico.

Se justifica realizar nuevas investigaciones tomando en consideración otras universidades que permitan generalizar los resultados para establecer planes de mejoramiento al interior de las instituciones

\section{BIBLIOGRAFÍA}

Bogoya M. (2012). Calidad de la educación superior. Facultad de Ciencias Naturales e Ingeniería, Universidad de Bogotá Jorge Tadeo Lozano. Informe edición 2011. Recuperado de: http://danielbogoya.utadeo.edu.co.

Briones, G. (2002). Metodología de la investigación cuantitativa en las ciencias sociales. Módulos de Investigación Social. ICFES. Arfo Editores. ISBN 9589329098 
Cervini, R. (2005). La equidad en la distribución de los logros de aprendizaje en algunos países de América Latina: A propósito de los resultados del LLECE. Sociedad Argentina de estudios comparados en educación. Recuperado de: http://www.google.com.co/url?sa=t\&rct=j\& $\mathrm{q}=\&$ esrc $=\mathrm{s} \&$ source $=$ web $\& \mathrm{~cd}=9 \& \mathrm{ved}=0 \mathrm{CGk}$ QFjAI\&url=http\%3A\%2F\%2Fwww.saece.o rg.ar\%2Fdocs $\% 2$ Fcongreso $1 \% 2 \mathrm{FCervini.do}$ c\&ei=F72GUeWTHqyt0AGSnoEg\&usg=A FQjCNEO0W9ECNYfTrrskom9zy0PuMdn bQ.

Chica, S; Galvis, D y Ramírez, A (2009). Determinantes del rendimiento académico en Colombia: pruebas ICFES Saber $11^{\circ}$. Recuperado de: http://repository.eafit.edu.co/bitstream/1078 4/580/1/2011-

5\%20Andres\%20Ramirez\%20Hassan.pdf.

Coleman, J. S. et al (1966). Equality of Educational Opportunity. Washington: US Government Printing Office.

Denegri, M., Del Vallle, C.,Gempp, R y Lara, M. (2006). Educación económica en la escuela: Hacia una propuesta de intervención. Recuperado de: http://www.scielo.cl/scielo.php?script=sci_a rttext\&pid=S0718 07052006000200006\&lng=es\&nrm=iso > . ISSN 0718-0705. doi: 10.4067/S071807052006000200006 .

Díez, E. y Díez F., (2005). Un modelo para la medición de la eficiencia en los departamentos universitarios. Recuperado de: http://institucional.us.es/revistas/universitari a/25/01\%20diez.pdf
Hernández, S., (2010). Metodología de la Investigación. McGrawHill.http://www.upcomillas.es/personal/pete r/investigacion/Dise\%F1osMedias.pdf.

Recuperado

ICFES. Pruebas Saber Pro. http://www.icfes.gov.co/examenes/saberpro.

ICFES, (2011). Competencias para la Educación Superior en Colombia. Recuperado de: http://www.ascofapsi.org.co/documentos/20 11/MEN_Competencias_Genericas.pdf

Jencks, C. S. (1972). "Inequality: a Reassessment of the Effect of Family and Schooling in

\section{Books. \\ America". En: New York: Basic}

Martínez, R. Heredia, Y. (2010). Tecnología educativa en el salón de clase: estudio retrospectivo de su impacto en el desempeño académico de estudiantes universitarios del área de Informática. , vol.15, n.45, pp. 371-390. ISSN 1405-6666. Recuperado de:http://www.scielo.org.mx/pdf/rmie/v15n4 5/v15n45a3.pdf.

Morales, P (2012). Diseños que se pueden analizar mediante el contraste de medias. Recuperado de: http://www.upcomillas.es/personal/peter/inv estigacion/Dise\%F1osMedias.pdf.

Riffo, B, Benotil, C. (2007). Procesamiento de oraciones con incrustación central y derecha en adultos jóvenes y adultos mayores. Recuperado de: http://www.scielo.cl/scielo.php?script=sci_a rttext\&pid=S0718$48832007000100002 \& \operatorname{lng}=$ en $\& n r m=i s o>$. ISSN 0718-4883. doi: $10.4067 / S 0718-$ 48832007000100002. 
Rivero, D, Vega, B y Balagué, J (2005). La medición del capital intelectual en las Universidades: Un modelo para potenciar su aportación a la sociedad. Recuperado de:http://pdfs.wke.es/2/4/6/6/pd0000012466. pdf.

Rizzo, M, (s.f). Mediciones para la Calidad Educativa a nivel internacional en Instituciones deEducación Superior. Construcción de Indicadores: $\mathrm{Su}$ interpretación y aplicación en procesos de evaluación y mejora de la calidad educativa. Recuperado de:http://www.udesa.edu.ar/files/MaeEduca cion/Microsoft $\% 20$ Word $\% 20$ \%20Rizzo\%20-\%20Resumen.pdf.

Universidad Nacional Abierta y a Distancia UNAD. (2011). Análisis del rendimiento saber Pro. Vicerrectoría Académica y de Investigación.

Universidad Nacional de Colombia. (2012). Análisis de los resultados obtenidos por la Universidad Nacional de Colombia sede Bogotá en las pruebas Saber Pro. Recuperado de: http://www.unal.edu.co/diracad/evaluacion/ Saber-Pro_2012/analisis_de_resultados.pdf 